Article

\title{
Enzymatic Synthesis of Novel Glycyrrhizic Acid Glucosides Using a Promiscuous Bacillus Glycosyltransferase
}

\author{
Longhai Dai ${ }^{1,2,+}$, Jiao Li ${ }^{1,3,+}$, Jiangang Yang ${ }^{1}$, Yan Men ${ }^{1}$, Yan Zeng ${ }^{1}$, Yi Cai ${ }^{1}$ and \\ Yuanxia Sun 1 ,* \\ 1 National Engineering Laboratory for Industrial Enzymes, Tianjin Institute of Industrial Biotechnology, \\ Chinese Academy of Sciences, Tianjin 300308, China; dai_lh@tib.cas.cn (L.D.); li_j1@tib.cas.cn (J.L.); \\ yang_jg1@tib.cas.cn (J.Y.); men_y@tib.cas.cn (Y.M.); Zeng_y@tib.cas.cn (Y.Z.); cai_y@tib.cas.cn (Y.C.) \\ 2 State Key Laboratory of Biocatalysis and Enzyme Engineering, School of Life Sciences, Hubei University, \\ Wuhan 430062, China \\ 3 University of Chinese Academy of Sciences, Beijing 100049, China \\ * Correspondence: sun_yx@tib.cas.cn; Tel.: +86-228-486-1960 \\ + These authors contributed equally to this work.
}

Received: 8 November 2018; Accepted: 27 November 2018; Published: 4 December 2018

\begin{abstract}
Glycyrrhetinic acid (GA) and glycyrrhizin (GA-3-O-[ $\beta$-D-glucuronopyranosyl-( $1 \rightarrow 2$ ) - $\beta$-D-glucuronopyranoside], GL) are the major bioactive components of Glycyrrhiza uralensis and possess multifarious notable biological activities. UDP-glycosyltransferase (UGT)-catalyzed glycosylation remarkably extends the structural and functional diversification of GA-glycoside derivatives. In this study, six glucosides (1-6) of GA and GL were synthesized using a Bacillus subtilis 168-originated flexible UDP-glycosyltransferase Bs-YjiC. Bs-YjiC could transfer a glucosyl moiety from UDP-glucose to the free C3 hydroxyl and/or C30 carboxyl groups of GA and GL and further elongate the C30 glucosyl chain via a $\beta$-1-2-glycosidic bond. Glycosylation significantly increased the water solubility of these novel glucosides by 4-90 folds. In vitro assays showed that GA monoglucosides ( $\mathbf{1}$ and $\mathbf{2}$ ) showed stronger antiproliferative activity against human liver cancer cells HepG2 and breast cancer cells MCF-7 than that of GL and GL glucosides. These findings provide significant insights into the important role of promiscuous UGTs for the enzymatic synthesis of novel bioactive GA derivatives.
\end{abstract}

Keywords: glycyrrhetinic acid; glycyrrhizin; Bacillus UDP-glycosyltransferase; glycosylation; cytotoxicity

\section{Introduction}

Triterpenoid saponins represent one of the largest groups of natural products with a structure consisting of a hydrophobic triterpene backbone and one or more sugar moieties attached to the hydroxyl and/or carboxyl groups through a $\beta$-glycosidic linkage $[1,2]$. These molecules are widely distributed in higher plants and confer protective functions to the plants due to their anti-insect, antimicrobial, and anti-palatable activities [3]. Triterpenoid saponins are also the major bioactive compounds of many important traditional medicinal plants, including Glycyrrhiza uralensis, Panax ginseng, Saponaria vaccaria, Bupleurum falcatum, and Siraitia grosvenorii [4-8]. These natural products exhibit a wide range of notable biological actions, including anticancer, antifatigue, anti-inflammatory, antidiabetic, antiaging, antihypertensive, and immune-potentiating properties [9].

Glycyrrhetinic acid (GA) and glycyrrhizin (GA-3-O-[ $\beta$-D-glucuronopyranosyl- $(1 \rightarrow 2)-\beta$-Dglucuronopyranoside], GL) are oleanane-type pentacyclic triterpenes. These compounds are the 
principal bioactive constituents of G. uralensis, which is a famous medicinal and dietary herbal plant recorded in the pharmacopoeias of many Asian countries [10]. GA possesses a variety of remarkable bioactivities, including anti-inflammatory, antibacterial, and antiviral effects [11,12]. GL is the most representative glycosylated derivative of GA and generally recognized as an indicator to access the quality of G. uralensis [13]. The attachment of two glucuronyl moieties to the C3 hydroxyl group of GA not only enhances its solubility but also endows GL with high-potency sweetness [14]. Most importantly, GL exhibits various special pharmacological activities, including neuroprotective, immunomodulatory, hepatoprotective, anti-asthma, anti-diabetes, and anti-cancer activities [15]. Clinically, GL has been widely used for the treatment of chronic hepatitis, hepatic carcinoma, liver cirrhosis, severe acute respiratory syndrome-associated coronavirus, and human immunodeficiency virus infections [16,17].

UDP-glycosyltransferase (UGT)-catalyzed glycosylation remarkably extends the structural and functional diversification of GA- glycoside derivatives [18]. Currently, only four UGTs involved in glycosylation of GA and GL have been functionally characterized, including GuUGAT and UGT73F17 from G. uralensis, UGT73C11 from Barbarea vulgaris, and YjiC1 from Bacillus subtilis CCTCC AB2012913 [19-22]. GuUGAT catalyzed a continuous two-step glucuronosylation at the C3 hydroxyl group of GA to synthesize GL, whereas UGT73F17 selectively catalyzed esterification at the C30 carboxyl group of GA and GL. UGT73C11 can regiospecifically transfer a glucosyl moiety from UDP-glucose (UDPG) to the free C3 hydroxyl group of GA. YjiC1 was also demonstrated to glycosylate the free C3 hydroxyl group of GA with UDPG as a sugar donor. Microbial UGTs are generally recognized as effective tools for glycodiversification of natural products due to their broad acceptor tolerance [23]. Hence, we aim to seek microbial UGTs for biosynthesis of novel GA glycosides.

Bs-YjiC from Bacillus subtilis 168 is a flexible and robust UGT that can glycosylate structurally diverse natural products [24-26]. In the present study, novel glucosides of GA and GL were synthesized using Bs-YjiC as a biocatalyst. The regio- and stereospecificity of Bs-YjiC toward GA and GL were elucidated by extensive structural analysis of the glycosylated derivatives using 1D NMR and 2D NMR spectra. In addition, the water solubility and in vitro cytotoxicity of these unnatural glucosides against human liver cancer cells HepG2 and breast cancer cells MCF-7 were also characterized.

\section{Results and Discussion}

\subsection{Glycosylation of $G A$ with Bs-YjiC}

The recombinant Bs-YjiC was expressed in E. coli BL21 (DE3) and purified by one-step nickel affinity chromatography (Supplementary Information Figure S1). GA is the common aglycone of GL and the other triterpenoid saponins in G. uralensis [1]. Thus, Bs-YjiC was preliminarily used to catalyze the in vitro glycosylation of GA with UDPG as a sugar donor (Figure 1). Four new products (1-4) from the Bs-YjiC-catalyzed reactants were identified by HPLC, whereas no new products were detected in the control reactants catalyzed by crude extracts prepared from E. coli BL21 (DE3) expressing pET28a (Figure 2A). Further mass analysis of product $1\left([\mathrm{M}+\mathrm{H}]^{+} \mathrm{m} / z 633.3967\right)$, product $2\left([\mathrm{M}+\mathrm{H}]^{+} \mathrm{m} / z\right.$ 633.3986), product $3\left([\mathrm{M}+\mathrm{H}]^{+} m / z\right.$ 795.4490), and product $4\left([\mathrm{M}+\mathrm{H}]^{+} m / z\right.$ 957.5030) confirmed that these novel products were monoglucoside, diglucoside, and triglucoside derivatives of $\mathrm{GA}\left(\mathrm{C}_{30} \mathrm{H}_{46} \mathrm{O}_{4}\right.$, calculated molecular weight, $[\mathrm{M}+\mathrm{H}]^{+} m / z$ 471.3468) (Figure 2B). Microbial UGTs are generally flexible toward both the aglycones and sugar donors [27]. Thus, UDP-GlcNAc, UDP-Gal, and UDP-GlcA were also tested as possible sugar donors under identical conditions as those of GA and UDPG. Bs-YjiC was only able to catalyze UDP-Gal with low activity (approximately $9 \%$ relative activity against the reaction using UDPG as a sugar donor), whereas activity of Bs-YjiC toward UDP-GlcA and UDP-GlcNAc was not detected (Supplementary Information Figure S2). These results indicated that Bs-YjiC favorably used UDPG as a sugar donor and could distinguish the sugar moiety of UDP-sugars. 


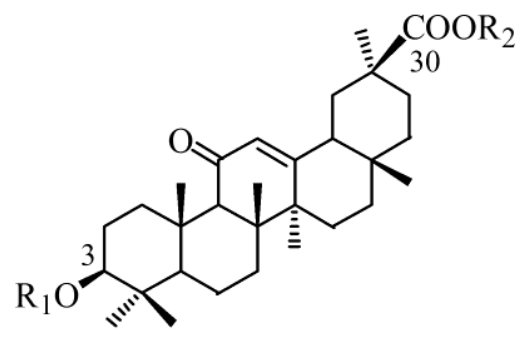

$\begin{array}{ccc}\text { Products } & \mathrm{R}_{1} & \mathrm{R}_{2} \\ \text { GA } & -\mathrm{H} & -\mathrm{H} \\ \mathbf{G L} & -\mathrm{GlcA}(2-1) \mathrm{GlcA} & -\mathrm{H} \\ \mathbf{1} & -\mathrm{H} & -\mathrm{Glc} \\ \mathbf{2} & -\mathrm{Glc} & -\mathrm{H} \\ \mathbf{3} & -\mathrm{Glc} & -\mathrm{Glc} \\ \mathbf{4} & -\mathrm{Glc} & -\mathrm{Glc}(2-1) \mathrm{Glc} \\ \mathbf{5} & -\mathrm{GlcA}(2-1) \mathrm{GlcA} & -\mathrm{Glc} \\ \mathbf{6} & -\mathrm{GlcA}(2-1) \mathrm{GlcA} & -\mathrm{Glc}(2-1) \mathrm{Glc}\end{array}$

Figure 1. Structures of GA, GL and the respective glycosylated products. Glc and GlcA represented the glucose and glucuronic acid moiety, respectively.
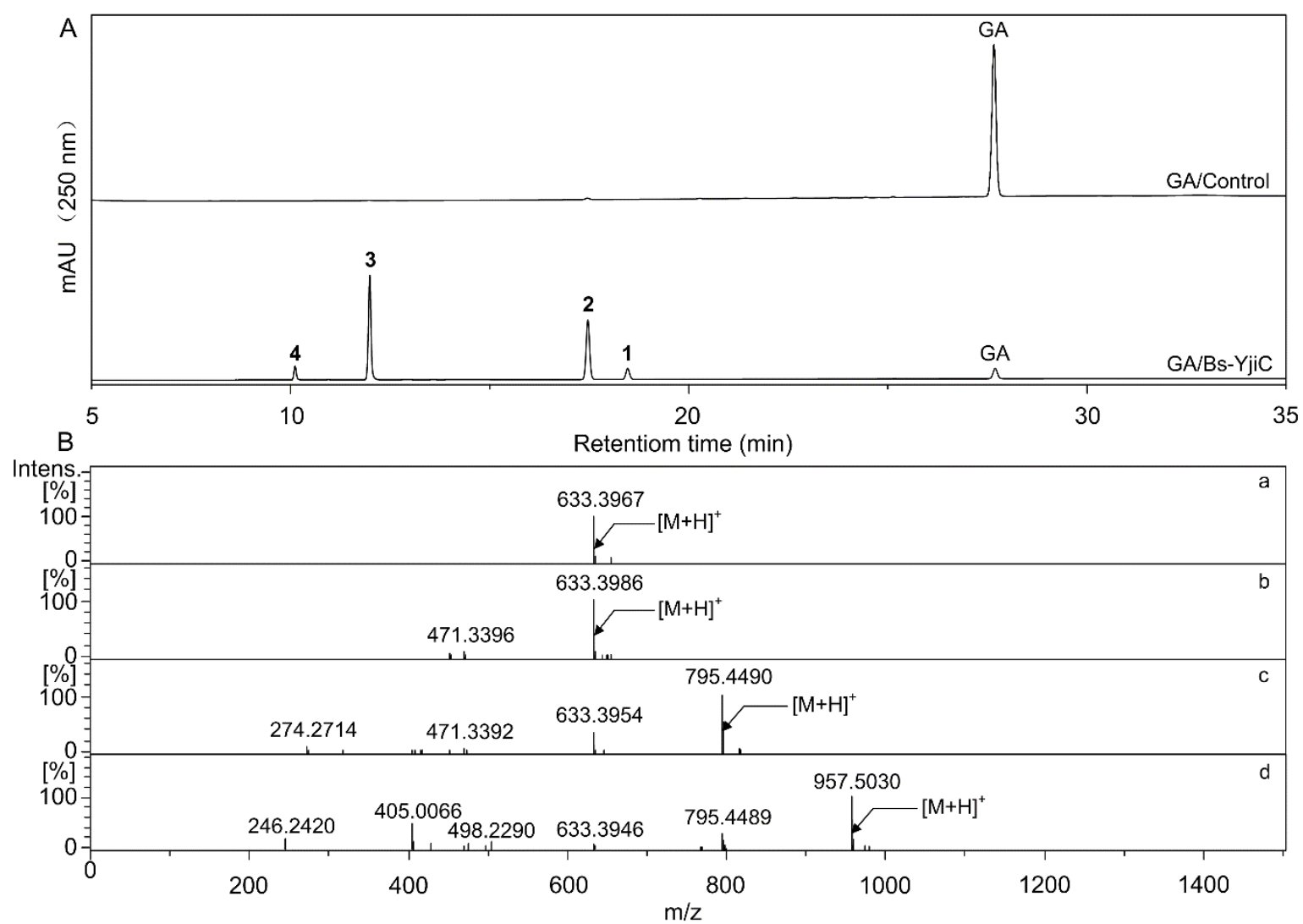

Figure 2. HPLC-ESI-MS analysis of the glycosylated products of GA. (A) HPLC chromatogram of Bs-YjiC-catalyzed reaction using GA as the aglycone. (B) MS spectra for products $\mathbf{1}$ (a), 2 (b), 3 (c), and $4(\mathrm{~d})$.

To elucidate the regio- and stereospecificity of Bs-YjiC toward GA, products 1-4 were purified and their structures were confirmed by NMR analyse (Supplementary Information Figures S3-S22). For monoglucoside 1, the anomeric carbon signal $\mathrm{C} 1^{\prime}\left(\delta_{\mathrm{C}} 95.6\right)$ shifted to higher field $(\sim 11.0 \mathrm{ppm})$ and the anomeric proton signal $\mathrm{H}^{\prime}\left(\delta_{\mathrm{H}} 5.52\right)$ shifted to lower field $(\sim 1.2 \mathrm{ppm})$ than the corresponding 
signals in an alcoholic glycosidic bond, suggesting that the glucosyl moiety was esterified with the C30 carboxyl group of GA (Supplementary Information Table S1) [28]. Furthermore, the HMBC correlations of the anomeric proton signal $\mathrm{H} 1^{\prime}\left(\delta_{\mathrm{H}} 5.52, \mathrm{~d}, J=8.20 \mathrm{~Hz}\right)$ with $\mathrm{C} 30\left(\delta_{\mathrm{C}} 176.7\right)$ of GA backbone reconfirmed that product 1 was GA-30-O- $\beta$-D-glucopyranoside. For monoglucoside 2, a notable downfield ${ }^{13} \mathrm{C}$-shift ( 11.2 ppm, "glycosylation shift") at C3 ( $\left.\delta_{\mathrm{C}} 90.4\right)$ indicated that a glucosyl moiety was attached to the C3 hydroxyl group of GA (Supplementary Information Table S2) [29]. The HMBC correlations between the anomeric proton signal $\mathrm{H}^{\prime}\left(\delta_{\mathrm{H}} 4.32, \mathrm{~d}, J=7.80 \mathrm{~Hz}\right)$ with $\mathrm{C} 3\left(\delta_{\mathrm{C}} 90.4\right)$ suggested that product 2 was GA-3-O- $\beta$-D-glucopyranoside. Diglucoside 3 exhibited spectroscopic data similar to those of products 1 and $\mathbf{2}$ (Supplementary Information Table S3). The observation of significant downfield shift of C3 ( 11.1 ppm) of GA backbone and upfield shift of the anomeric carbon signal C1" ( $11.0 \mathrm{ppm}$ ) suggested that a glucosyl moiety was attached to the $\mathrm{C} 3$ hydroxyl and $\mathrm{C} 30$ carboxyl groups of GA, respectively. The HMBC correlations of the anomeric proton signal $\mathrm{H}^{\prime}\left(\delta_{\mathrm{H}} 4.32, \mathrm{~d}, J=7.80 \mathrm{~Hz}\right)$ with $\mathrm{C} 3\left(\delta_{\mathrm{C}} 90.3\right)$ and the anomeric proton signal H1 $1^{\prime \prime}\left(\delta_{\mathrm{H}} 5.52, \mathrm{~d}, J=8.20 \mathrm{~Hz}\right)$ with $\mathrm{C} 30\left(\delta_{\mathrm{C}} 176.7\right)$ further confirmed that product 3 was GA-3-O- $\beta$-D-glucopyranosyl-30-O- $\beta$-D-glucopyranoside. ${ }^{1} \mathrm{H}$ NMR and ${ }^{13} \mathrm{C}$ NMR spectra of triglucoside 4 were highly similar to those of product 3 except for the glucosyl chain unit (Supplementary Information Table S4). The presence of three glucosyl moieties was confirmed by the anomeric proton signals, including $\mathrm{H}^{\prime}\left(\delta_{\mathrm{H}} 4.32, \mathrm{~d}, J=7.80 \mathrm{~Hz}\right), \mathrm{H} 1^{\prime \prime}\left(\delta_{\mathrm{H}} 5.57\right.$, $\mathrm{d}, J=7.80 \mathrm{~Hz})$, and $\mathrm{H} 1^{\prime \prime \prime}\left(\delta_{\mathrm{H}} 4.76, \mathrm{~d}, J=7.80 \mathrm{~Hz}\right)$. In the COSY spectra, the sugar anomeric carbon signal $\left(\delta_{\mathrm{C}} 79.0\right)$ was assigned to the $\mathrm{C}^{\prime \prime}$ of the 30-O-inner glucosyl moiety. By comparison of the ${ }^{1} \mathrm{H}$ NMR and ${ }^{13} \mathrm{C}$ NMR spectra of product 4 with those of product 3 , significant glycosylation shifts were observed for $\mathrm{C}^{\prime \prime}(-1.7 \mathrm{ppm}), \mathrm{C}^{\prime \prime}$ ( $\left.+5.0 \mathrm{ppm}\right)$, and $\mathrm{C}^{\prime \prime}$ (+0.3 ppm) of the 30-O-inner glucosyl moiety, indicating that the outer glucosyl moiety was attached to the 30-O-inner glucosyl moiety by a $\beta-1-2-$ glycosidic bond. In addition, the identification of $\mathrm{HMBC}$ correlations between $\mathrm{H}^{\prime \prime \prime}\left(\delta_{\mathrm{H}} 4.76\right.$, d, $J=7.80 \mathrm{~Hz})$ with $\mathrm{C}^{\prime \prime}\left(\delta_{\mathrm{C}} 79.0\right)$ reconfirmed the outer glucosyl moiety was attached to the $\mathrm{C}^{\prime \prime}$ position of the 30-O-inner glucosyl moiety. On the basis of these data, product 4 was identified as GA-3-O- $\beta$-D-glucopyranosyl-30-O-[ $\beta$-D-glucuronopyranosyl-( $1 \rightarrow 2)-O-\beta$-D-glucuronopyranoside]. The coupling constants of the anomeric protons $(J=7.80-8.20 \mathrm{~Hz})$ of products $1-4$ indicated that all the glucosyl moieties adopted the $\beta$-configuration, which was consistent with the inverting mechanism of UGTs [30]. All the four glucosides (1-4) of GA were unnatural products, and products 3 and 4 were synthesized in this study for the first time. Thus, Bs-YjiC from B. subtilis 168 was the first reported UGT that could transfer a glucosyl moiety from UDPG to the free C3 hydroxyl and/or C30 carboxyl groups of GA and further elongate the C30 glucosyl chain via a $\beta$-1-2-glycosidic bond. Glycosylation is the prominent biological mechanism for structural and functional diversity of triterpene saponins [2]. Thus, unnatural products 1-4 synthesized in this study should also possess biological and pharmacological activities that are the same as those as other natural GA glycosides that are usually decorated with glucuronyl, xylosyl, or rhamnosyl moieties.

\subsection{Glycosylation of $G L$ with Bs-YjiC}

GL is the most representative glycoside of GA (Figure 1), which contains a free C30 carboxyl group and two glucuronyl moieties attached to the $\mathrm{C} 3$ hydroxyl via a $\beta$-1-2-glycosidic bond [31]. Therefore, a reaction by incubation of GL with UDPG was further performed under conditions identical to those of GA. Two new products ( 5 and $\mathbf{6}$ ) from the Bs-YjiC-catalyzed reaction mixtures were identified by HPLC (Figure 3A). Mass analysis of product 5 ([M $\left.+\mathrm{H}]^{+} m / z^{+} \sim 985.4630\right]^{+},[\mathrm{M}+\mathrm{H}-\mathrm{Glc}]^{+} m / z$ 823.3983, and $[\mathrm{M}+\mathrm{H}-\mathrm{Glc}-2 \mathrm{GlcA}]^{+} m / z$ 471.3386), and product $6\left([\mathrm{M}+\mathrm{H}]^{+} m / z\right.$ 1147.5150, $[\mathrm{M}+\mathrm{H}-\mathrm{Glc}]^{+} m / z$ 985.4600, [M + H-2GlcA $]^{+} m / z$ 823.3989, [M + H-2Glc-GlcA $]^{+} m / z$ 647.3764, and [M + H-2Glc-2GlcA ${ }^{+}$ $m / z$ 471.3380) confirmed that they were monoglucoside and diglucoside derivatives of $\mathrm{GL}\left(\mathrm{C}_{42} \mathrm{H}_{62} \mathrm{O}_{16}\right.$, calculated molecular weight, $[\mathrm{M}+\mathrm{H}]^{+} m / z$ 823.3980), respectively (Figure 3B). GL only contains a free C30 carboxyl group. Thus, Bs-YijC could also elongate the sugar chain of GL through iterative glycosylation in the same way as using GA as the aglycone. 

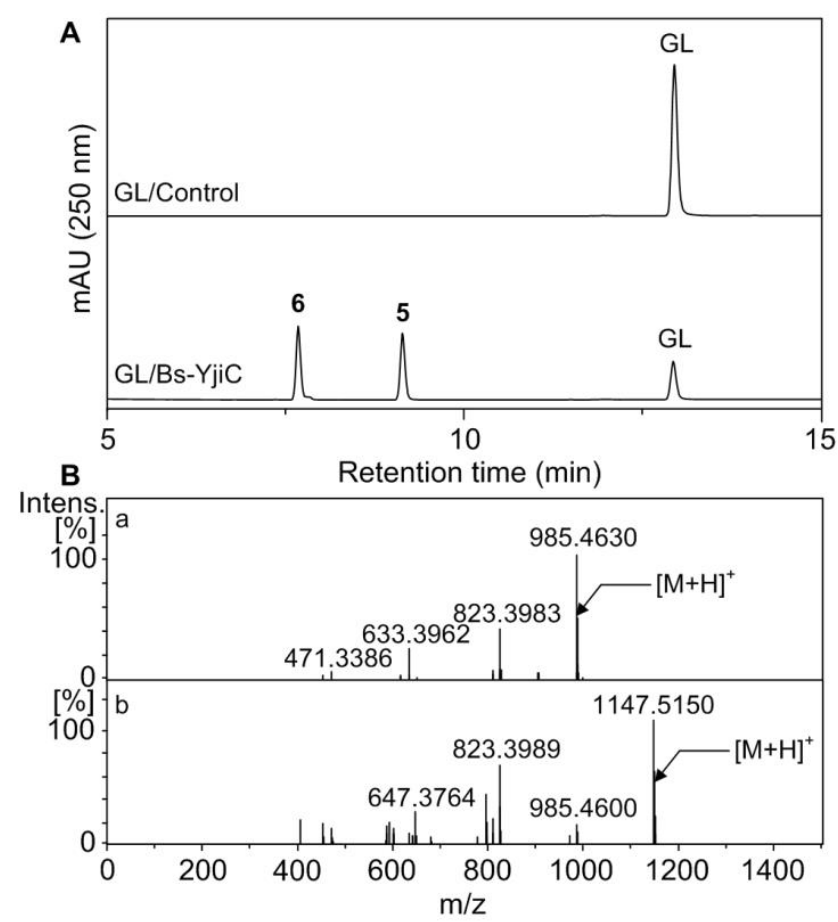

Figure 3. HPLC-ESI-MS analysis of the glycosylated products of GL. (A) HPLC chromatograms of Bs-YjiC-catalyzed reaction using GA as the aglycone. (B) MS spectra for products 5 (a) and $\mathbf{6}$ (b).

${ }^{1} \mathrm{H}$ NMR and ${ }^{13} \mathrm{C}$ NMR spectra of product 5 were highly similar to those of products 1,3 , and GL (Supplementary Information Figures S23-S27) (Supplementary Information Table S5). ${ }^{1} \mathrm{H}$ NMR spectra of product 5 indicated the presence of three sugar anomeric proton signals $\left(\mathrm{H}^{\prime}, \delta_{\mathrm{H}} 4.52\right.$, $\mathrm{d}, J=7.80 \mathrm{~Hz} ; \mathrm{H}^{\prime \prime}, \delta_{\mathrm{H}} 4.63, \mathrm{~d}, J=7.80 \mathrm{~Hz} ; \mathrm{H}^{\prime \prime \prime}, \delta_{\mathrm{H}} 5.52, \mathrm{~d}, J=8.20 \mathrm{~Hz}$ ), which was consistent with the MS spectra that GL was decorated with one more glucosyl moiety. The sugar anomeric carbon signal $\mathrm{C1}^{\prime \prime \prime}\left(\delta_{\mathrm{C}} 95.5\right)$ shifted to higher field $(\sim 11.0 \mathrm{ppm})$ and the sugar anomeric carbon signal $\mathrm{H}^{\prime \prime \prime}\left(\delta_{\mathrm{H}} 5.52\right)$ shifted to lower field $(\sim 1.3 \mathrm{ppm})$ suggested that the glucosyl moiety was esterified with the C30 carboxyl group of GL. Furthermore, the HMBC correlations of the sugar anomeric signal H1 $1^{\prime \prime \prime}\left(\delta_{\mathrm{H}} 5.52, \mathrm{~d}, J=8.20 \mathrm{~Hz}\right)$ with $\mathrm{C} 30\left(\delta_{\mathrm{C}} 176.7\right)$ reconfirmed that product 5 was GL-30-O- $\beta$-D-glucopyranoside (Licorice saponin A3). ${ }^{1} \mathrm{H}$ NMR and ${ }^{13} \mathrm{C}$ NMR spectra of product 6 were highly similar to those of products 4, 5, and GL (Supplementary Information Figures S28-S32) (Supplementary Information Table S6). In the COSY spectra, signals at $\delta_{\mathrm{C}} 94.0$ and $\delta_{\mathrm{C}} 79.2$ were assigned to the $\mathrm{C}^{\prime \prime \prime}$ and $\mathrm{C}^{\prime \prime \prime}$ of the 30-O-inner glucosyl moiety. The observation of significant upfield shift of $\mathrm{C1}^{\prime \prime \prime}$ ( 12.6 ppm, "glycosylation shift") was consistent with the formation of ester-linked glycosidic bond between the 30-O-inner glucosyl moiety and C30 carboxyl group of GA backbone. By comparison of the ${ }^{1} \mathrm{H}$ NMR and ${ }^{13} \mathrm{C}$ NMR spectra of products 5 and $\mathbf{6}$, significant glycosylation shifts were also observed for $\mathrm{C}^{\prime \prime \prime}(-1.5 \mathrm{ppm}), \mathrm{C}^{\prime \prime \prime}(+5.3 \mathrm{ppm})$, and $\mathrm{C}^{\prime \prime \prime}(-0.6)$ of the 30-O-inner glucosyl moiety, suggesting that the 30-O-outer glucosyl moiety was attached to the 30-O-inner glucosyl moiety by a $\beta$-1-2-glycosidic bond. In the HMBC, long-range correlations between the sugar anomeric carbon signal $\mathrm{H}-1^{\prime \prime \prime \prime}\left(\delta_{\mathrm{H}} 4.75, \mathrm{~d}, J=7.80 \mathrm{~Hz}\right)$ of the $30-O$-outer glucosyl moiety with $\mathrm{C} 2^{\prime \prime \prime}\left(\delta_{\mathrm{C}} 79.2\right)$ of the 30-O-inner glucosyl moiety further confirmed the outer glucosyl moiety was attached to the $\mathrm{C} 2$ position of the 30-O-inner glucosyl moiety. Based on these spectra data, product 6 was identified as GL-30-O-[ $\beta$-D-glucuronopyranosyl-( $1 \rightarrow 2)-O-\beta$-D-glucopyranoside], which was an unnatural product and was synthesized for the first time in this study. Typically, UGTs belong to the GT-B type superfamily with an N-terminal aglycone-binding domain and a C-terminal UDP-sugar-binding domain [32]. Bs-YjiC could transfer a glucosyl moiety to the free C30 carboxyl group of GL and further elongate the sugar chain by a $\beta-1-2$-glycosidic bond to synthesize product $6\left(\mathrm{C}_{54} \mathrm{H}_{82} \mathrm{O}_{26},[\mathrm{M}+\mathrm{H}]^{+} m / z\right.$ 1147.5150), 
indicating that the acceptor binding pocket of Bs-YjiC should be considerably open and large. Further structural analysis of Bs-YjiC should be carried out to elucidate its structure-function relationship.

\subsection{Kinetic Parameters of Bs-YjiC toward GA and GL}

To evaluate the affinity and catalytic efficiency of Bs-YjiC, the kinetic parameters of Bs-YjiC toward GA and GL were determined by nonlinear-regression analysis (Supplementary Information Figure S33). The Michaelis constant $\left(K_{\mathrm{m}}\right)$ value of Bs-YjiC toward GA $(245.00 \mu \mathrm{M})$ was about 5-fold higher than that of Bs-YjiC toward GL $(58.73 \mu \mathrm{M})$ (Table 1$)$, indicating that the affinity of Bs-YjiC against GL was much higher than that of Bs-YjiC against GA. However, the turn over number $\left(k_{\text {cat }}\right)$ of Bs-YjiC against GA $\left(3.36 \mathrm{~s}^{-1}\right)$ was approximately 11 -fold higher than that of GL $\left(0.31 \mathrm{~s}^{-1}\right)$. Consequently, the catalytic efficiency $\left(k_{\text {cat }} / K_{\mathrm{m}}\right)$ of Bs-YjiC against GA $\left(1.37 \times 10^{4} \mathrm{~s}^{-1} \mathrm{M}^{-1}\right)$ was about 2-fold higher than that of Bs-YjiC against GL $\left(0.53 \times 10^{4} \mathrm{~s}^{-1} \mathrm{M}^{-1}\right)$. Compared with the kinetic parameters of B. vulgaris UGT73C11 and G. uralensis UGT73F17 [20,22], the catalytic efficiencies of Bs-YjiC against GA and GL were considerably high, indicating that Bs-YjiC was an effective biocatalyst for glycosylation of GA and GL. Currently, all the key genes involved in the biosynthetic pathway of GA have been cloned and functionally characterized [33,34]. An engineered yeast cell factory that can produce substantial amounts of GA has recently been constructed by introducing the whole biosynthetic pathway of GA into S. cerevisiae [8]. Thus, it will be of particular interest to introduce Bs-YjiC or an engineered Bs-YjiC into the GA-producing chassis cell factory to synthesize these unnatural GA glucosides or a specific GA glucoside via protein engineering, synthetic biology, and metabolic engineering.

Table 1. Kinetic parameters of Bs-YjiC toward GA and GL.

\begin{tabular}{cccc}
\hline Substrate & $\boldsymbol{K}_{\mathrm{m}}(\boldsymbol{\mu M})$ & $\boldsymbol{k}_{\text {cat }}\left(\mathbf{s}^{-\mathbf{1}}\right)$ & $\boldsymbol{k}_{\text {cat }} / \boldsymbol{K}_{\mathrm{m}}\left(\mathbf{s}^{-\mathbf{1}} \mathbf{M}^{-\mathbf{1}}\right)$ \\
\hline GA & $245.00 \pm 23.03$ & $3.36 \pm 0.13$ & $1.37 \times 10^{4}$ \\
GL & $58.73 \pm 4.07$ & $0.31 \pm 0.01$ & $0.53 \times 10^{4}$ \\
\hline
\end{tabular}

\subsection{Solubility of the Glycosylated Products}

The poor solubility of natural products resulted in a short retention time as well as a low absorption in the intestine [35,36]. Glycosylation can be used to enhance the solubility of hydrophobic natural products and thus to improve their bioavailabilities and pharmacodynamics [37]. Therefore, the effect of Bs-YjiC-catalyzed glycosylation on water solubility of GA and GL glucosides was evaluated (Table 2). As could be expected, the attachment of glucosyl moieties to GA and GL significantly increased their water solubility. The solubility of GA was limited to $29 \mu \mathrm{M}$, whereas the corresponding glucosides could be dissolved to $58 \mu \mathrm{M}$ (product 1), $242 \mu \mathrm{M}$ (product 2), $2022 \mu \mathrm{M}$ (product 3), and $98326 \mu \mathrm{M}$ (product 4), respectively. Notably, products 1 and 2 were structural isomers; however, product 2 exhibited 4 times more soluble than that of product 1 . These results were in agreement with previous studies that glycosylation position influenced solubility $[38,39]$. Compared with the water solubility of GL $(1526 \mu \mathrm{M})$, the water solubility of product $5(136,698 \mu \mathrm{M})$ was significantly increased by 90 times after the C30 carboxyl of GL was decorated with only one glucosyl moiety.

Table 2. Water solubility of GA, GL and the glycosylated products $(\mu \mathrm{M})$.

\begin{tabular}{ccc}
\hline Compound & Solubility & Relative Solubility \\
\hline GA & $29 \pm 2$ & 1 \\
GL & $1526 \pm 322$ & 53 \\
$\mathbf{1}$ & $58 \pm 2$ & 2 \\
$\mathbf{2}$ & $242 \pm 20$ & 8 \\
$\mathbf{3}$ & $2022 \pm 192$ & 70 \\
$\mathbf{4}$ & $98,326 \pm 5022$ & 3390 \\
$\mathbf{5}$ & $136,698 \pm 8425$ & 4714 \\
$\mathbf{6}$ & $\mathrm{ND}$ & $\mathrm{ND}$ \\
\hline
\end{tabular}

ND represented that water solubility was not determined because we could not prepare enough product 6 . 


\subsection{In Vitro Cytotoxicity of the Glycosylated Products}

Sugar moieties appended to natural products not only enhance their solubility but also influence their pharmacological efficacies and/or molecular mechanisms of action [40]. GL as the most representative glycosylated product of GA has been clinically used as a hepatoprotective medicine for the treatment of chronic hepatitis, liver cirrhosis and hepatic carcinoma [12]. Thus, the bioactivity of all the novel GA and GL glucosides against human liver cancer cells HepG2 and breast cancer cells MCF-7 was evaluated and compared with that of GL (Table 3). Monoglucosides $\mathbf{1}$ and $\mathbf{2}$ and GL showed remarkable inhibitory effects on $\mathrm{HepG} 2$, with $\mathrm{IC}_{50}$ values ranged from 7.2 to $37.3 \mu \mathrm{M}$. Similarly, monoglucosides 1 and $\mathbf{2}$ and GL can also inhibit the proliferation of MCF-7 with IC $_{50}$ values of 7.7, 13.0, and $113.7 \mu \mathrm{M}$, respectively. However, products 3-6 were inactive toward both HepG2 and MCF-7 (cut-off $200 \mu \mathrm{M}$ ). In addition, both monoglucosides $\mathbf{1}$ and $\mathbf{2}$ exhibited stronger cytotoxicity against HepG2 and MCF-7 than those of GL. The present study was identical to the previous findings on GA-3-O- $\beta$-D-glucuronide (a hydrolyzed derivative of GL), which showed stronger inhibitory activity against diverse human cancer cells than that of GL [41,42]. Notably, products $\mathbf{1}$ and $\mathbf{2}$ were isomers of GA; however, product 1 exhibited stronger activity against HepG2 ( $\sim 3.5$ folds) and MCF-7 ( 1.7 folds) than those of product 2 , indicating that the binding position of the glucosyl moiety significantly influenced the cytotoxic activities of GA glucosides.

Table 3. In vitro cytotoxicity of products 1 and $2\left(\mathrm{IC}_{50}, \mu \mathrm{M}\right){ }^{\mathrm{a}}$

\begin{tabular}{ccc}
\cline { 2 - 3 } Compound & HepG2 & MCF-7 \\
\hline $\mathrm{GL}^{\mathrm{b}}$ & $37.3 \pm 2.5$ & $113.7 \pm 10.4$ \\
$\mathbf{1}$ & $7.2 \pm 0.6$ & $7.7 \pm 0.6$ \\
$\mathbf{2}$ & $25.4 \pm 1.6$ & $13.0 \pm 1.4$ \\
& a Products 3-6 were inactive $\left(\mathrm{IC}_{50}\right.$ values $\left.>200 \mu \mathrm{M}\right){ }^{\mathrm{b}}{ }^{\mathrm{G}}$ GL as a clinical anticancer drug was used as a positive control.
\end{tabular}

\section{Materials and Methods}

\subsection{Chemicals and Reagents}

GA and GL were purchased from Chengdu Biopurify Phytochemicals Co., Ltd. (Chengdu, China). UDPG, UDP-N-acetylglucosamine (UDP-GlcNAc), UDP-galactose (UDP-Gal), and UDP-glucuronic acid (UDP-GlcA) were obtained from Sigma-Aldrich (St. Louis, USA). Human liver cancer cells HepG2 and breast cancer cells MCF-7 preserved in our lab were grown in Dulbecco's modified Eagle's medium (GIBCO, MA, USA) supplemented with $10 \%$ heat-inactivated fetal bovine serum, $100 \mu \mathrm{g} \mathrm{mL}^{-1}$ streptomycin, and 100 units $\mathrm{mL}^{-1}$ penicillin [24].

\subsection{In Vitro Enzymatic Glycosylation}

Expression of Bs-YjiC (GenBank accession no. NP_389104) was performed as reported previously [24]. Recombinant Escherichia coli BL21 (DE3) cells harboring plasmid pET28a-Bs-YjiC were inoculated into Luria-Bertani medium supplemented with $50 \mathrm{mg} \mathrm{L}^{-1}$ kanamycin and cultured at $37^{\circ} \mathrm{C}$ and $200 \mathrm{rpm}$ until the $\mathrm{OD}_{600}$ reached approximately 0.8. The $\mathrm{N}$-terminal $\mathrm{His}_{6}$-tagged $\mathrm{Bs}-\mathrm{YjiC}$ was induced with $0.2 \mathrm{mM}$ isopropyl- $\beta$-D-thiogalactopyranoside at $16^{\circ} \mathrm{C}$ and $200 \mathrm{rpm}$ for $16-18 \mathrm{~h}$. The recombinant cells were harvested by centrifugation at $5500 \mathrm{rpm}$ for $10 \mathrm{~min}$ at $4{ }^{\circ} \mathrm{C}$ and resuspended in binding buffer ( $50 \mathrm{mM}$ Tris- $\mathrm{HCl}, \mathrm{pH} 8.0,150 \mathrm{mM} \mathrm{NaCl}$, and $25 \mathrm{mM}$ imidazole). After disrupted with a French press, the cell lysates were centrifuged at $15,000 \mathrm{rpm}$ for $60 \mathrm{~min}$ at $4{ }^{\circ} \mathrm{C}$. Then, the supernatant containing $\mathrm{N}$-terminal $\mathrm{His}_{6}$-tagged Bs-YjiC was purified by Ni-NTA agarose affinity column connected to an AKTA Purifier system (GE Healthcare, Chicago, IL, USA).

The glycosylation reaction was performed in $300 \mu \mathrm{L}$ reaction buffer ( $50 \mathrm{mM}$ Tris- $\mathrm{HCl}, \mathrm{pH} 8.0)$ containing $1 \mathrm{mM} \mathrm{GA}$ or GL, $5 \mathrm{mM}$ diverse UDP-sugars (UDPG, UDP-GlcA, UDP-Gal, or UDP-GlcNAc), $10 \mathrm{mM} \mathrm{MgCl}$, and $10 \mu \mathrm{g}$ purified Bs-YjiC. After incubation at $40{ }^{\circ} \mathrm{C}$ for $0.5 \mathrm{~h}$, the reactions were 
quenched by adding equal volume of methanol and then analyzed by high-performance liquid chromatography (HPLC) or HPLC coupled with quantitative time of flight-high-resolution electrospray ionization-mass spectrometry (HPLC-ESI-MS) as reported previously [29].

\subsection{Kinetic Parameters of Bs-YjiC}

Kinetic studies $(300 \mu \mathrm{L})$ of Bs-YjiC toward GA and GL were conducted with purified Bs-YjiC $(1 \mu \mathrm{g}$ for GA and $2 \mu \mathrm{g}$ for GL), $10 \%$ DMSO ( $v / v), 50 \mathrm{mM}$ Tris- $\mathrm{HCl}(\mathrm{pH} 8.0), 10 \mathrm{mM} \mathrm{MgCl} 2,10 \mathrm{mM}$ UDPG, and varying concentrations of GA $(75-800 \mu \mathrm{M})$ or GL $(12.5-300 \mu \mathrm{M})$. The reactions were incubated at $40{ }^{\circ} \mathrm{C}$ for $20 \mathrm{~min}$ and terminated by adding equal volume of methanol.

\subsection{Structural Analysis of GA and GL Glucosides}

Scale-up preparation (500 mL) of products 1-6 was described above. The glycosylated products were purified by preparative HPLC system coupled with a preparative reverse-phase C18 column $(21.2 \times 250 \mathrm{~mm}, 5 \mu \mathrm{m}$ particle, Welch, Shanghai, China). The preparative column was eluted with distilled water (solvent A) and methanol (solvent B) by using a gradient program of $40-100 \%$ B in 0-60 min. The purified fractions containing target GA or GL glucosides were pooled and concentrated by using the rotary evaporator and vacuum freeze drier. The dried powders were dissolved in methanol- $d_{4}$. NMR experiments were performed on a Bruker DMX600 NMR spectrometer (Bruker, Bremen, Germany).

\subsection{Water Solubility Tests}

Water solubility of GA, GL, and the respective glucosides was determined as previously described [42]. Excess amounts of the respective glycosylated products, GA, or GL were added in distilled water. The suspensions were extensively mixed by vortexed for $2 \mathrm{~h}$, equilibrated overnight at $25^{\circ} \mathrm{C}$, and centrifuged at $14,000 \mathrm{rpm}$ for $10 \mathrm{~min}$ at room temperature. The supernatants were diluted with appropriate volumes of methanol and tested with HPLC method described above.

\subsection{In Vitro Cytotoxic Assays}

In vitro cytotoxic activities of GA and GL glucosides against human liver cancer cells HepG2 and breast cancer cells MCF-7 were evaluated using the 3-(4,5-dimethylthiazol2-yl)-2,5-diphenyltetrazolium bromide (MTT) assay as reported in our previous study [43]. GL as the most representative glycosylated product of GA and a first-line anticancer drug for the treatment of hepatic carcinoma was used as a positive control.

\section{Conclusions}

In summary, Bs-YjiC from B. subtilis 168 was the first reported UGT that can glycosylate the C3 hydroxyl and/or C30 carboxyl of GA and GL and elongate the C30 glucosyl chain via a $\beta$-1-2-glycosidic bond. GA-30-O- $\beta$-D-glucopyranoside (1) and GA-3-O- $\beta$-D-glucopyranoside (2) displayed stronger cytotoxic activity against cancer cell lines than that of GL. In vivo anticancer activity of monoglucosides $\mathbf{1}$ and $\mathbf{2}$ should be studied further.

Supplementary Materials: The supplementary materials are available online at http:/ /www.mdpi.com/2073$4344 / 8 / 12 / 615 /$ s1. Table S1. ${ }^{1} \mathrm{H}$ - and ${ }^{13} \mathrm{C}-\mathrm{NMR}$ spectral data for product 1 (Methanol- $d_{4}, 600 \mathrm{MHz}$ ). Table S2. ${ }^{1} \mathrm{H}$ - and ${ }^{13} \mathrm{C}-\mathrm{NMR}$ spectral data for product 2 (Methanol- $d_{4}, 600 \mathrm{MHz}$ ). Table S3. ${ }^{1} \mathrm{H}$ - and ${ }^{13} \mathrm{C}-\mathrm{NMR}$ spectral data for product 3 (Methanol- $d_{4}, 600 \mathrm{MHz}$ ). Table S4. ${ }^{1} \mathrm{H}$ - and ${ }^{13} \mathrm{C}-\mathrm{NMR}$ spectral data for product 4 (Methanol- $d_{4}$, $600 \mathrm{MHz}$ ). Table S5. ${ }^{1} \mathrm{H}$ - and ${ }^{13} \mathrm{C}-\mathrm{NMR}$ spectral data for product 5 (Methanol- $d_{4}, 600 \mathrm{MHz}$ ). Table S6. ${ }^{1} \mathrm{H}-$ and ${ }^{13} \mathrm{C}-\mathrm{NMR}$ spectral data for product 6 (Methanol- $\left.d_{4}, 600 \mathrm{MHz}\right)$. Figure S1. SDS-PAGE analysis of purified Bs-YjiC. Figure S2. Assessment of sugar donors of Bs-YjiC using GA as the aglycon. Figure S3. ${ }^{1} \mathrm{H}$ NMR spectrum of product 1 (Methanol- $\left.d_{4}\right)$. Figure S4. ${ }^{13} \mathrm{C}$ NMR spectrum of product 1 (Methanol- $d_{4}$ ). Figure S5. HMBC spectrum of product 1 (Methanol- $\left.d_{4}\right)$. Figure S6. HSQC spectrum of product 1 (Methanol- $d_{4}$ ). Figure S7. COSY NMR spectrum of product $1\left(\right.$ Methanol- $\left.d_{4}\right)$. Figure S8. ${ }^{1} \mathrm{H}$ NMR spectrum of product 2 (Methanol- $\left.d_{4}\right)$. Figure S9. ${ }^{13} \mathrm{C}$ 
NMR spectrum of product 2 (Methanol- $d_{4}$ ). Figure S10. HMBC spectrum of product 2 (Methanol- $d_{4}$ ). Figure S11. HSQC NMR spectrum of product $2\left(\right.$ Methanol- $\left.d_{4}\right)$. Figure S12. COSY spectrum of product $2\left(\right.$ Methanol- $\left.d_{4}\right)$. Figure S13. ${ }^{1} \mathrm{H}$ NMR spectrum of product 3 (Methanol- $\left.d_{4}\right)$. Figure S14. ${ }^{13} \mathrm{C}$ NMR spectrum of product 3 $\left(\right.$ Methanol- $\left.d_{4}\right)$. Figure S15. HMBC spectrum of product 3 (Methanol- $\left.d_{4}\right)$. Figure S16. HSQC spectrum of product 3 $\left(\right.$ Methanol- $\left.d_{4}\right)$. Figure S17. COSY spectrum of product 3 (Methanol- $\left.d_{4}\right)$. Figure S18. ${ }^{1} \mathrm{H}$ NMR spectrum of product 4 (Methanol- $\left.d_{4}\right)$. Figure S19. ${ }^{13} \mathrm{C}$ NMR spectrum of product 4 (Methanol- $\left.d_{4}\right)$. Figure S20. HMBC spectrum of product 4 (Methanol- $\left.d_{4}\right)$. Figure S21. HSQC spectrum of product 4 (Methanol- $\left.d_{4}\right)$. Figure S22. COSY spectrum of product 4 (Methanol- $\left.d_{4}\right)$. Figure S23. ${ }^{1} \mathrm{H}$ NMR spectrum of product 5 (Methanol- $d_{4}$ ). Figure S24. ${ }^{13} \mathrm{C}$ NMR spectrum of product $5\left(\right.$ Methanol- $\left.d_{4}\right)$. Figure S25. HMBC spectrum of product 5 (Methanol- $d_{4}$ ). Figure S26. HSQC spectrum of product 5 (Methanol- $\left.d_{4}\right)$. Figure S27. COSY spectrum of product 5 (Methanol- $\left.d_{4}\right)$. Figure S28. ${ }^{1} \mathrm{H}$ NMR spectrum of product 6 (Methanol- $\left.d_{4}\right)$. Figure S29. ${ }^{13} \mathrm{C}$ NMR spectrum of product 6 (Methanol- $d_{4}$ ). Figure S30. HMBC spectrum of product $6\left(\right.$ Methanol- $\left.d_{4}\right)$. Figure S31. HSQC NMR spectrum of product $6\left(\right.$ Methanol- $\left.d_{4}\right)$. Figure S32. COSY spectrum of product 6 (Methanol- $\left.d_{4}\right)$. Figure S33. Kinetic Parameters of Bs-YjiC toward GA and GL calculated by nonlinear regression analysis using GraphPad Prism 5.0 software.

Author Contributions: L.D. and J.L. designed research; J.Y., Y.M. and Y.C. performed research; J.L., Y.Z. and Y.C. analyzed data; L.D. wrote the manuscript. Y.S. revised the manuscript.

Funding: This research was financially supported by grants from the National Natural Science Foundation of China (No. 31771909), the Key Research Program of the Chinese Academy of Sciences (No. KFZD-SW-215), and the Key Research Program of Jiangxi (No.20171ACH80014).

Conflicts of Interest: The authors declare no conflict of interest.

\section{References}

1. Seki, H.H.; Tamura, K.; Muranaka, T. P450s and UGTs: Key players in the structural diversity of triterpenoid saponins. Plant Cell Physiol. 2015, 56, 1463. [CrossRef] [PubMed]

2. Shibuya, M.; Nishimura, K.; Yasuyama, N.; Ebizuka, Y. Identification and characterization of glycosyltransferases involved in the biosynthesis of soyasaponin I in Glycine Max. FEBS Lett. 2010, 584, 2258-2264. [CrossRef] [PubMed]

3. Naoumkina, M.A.; Dixon, R.A. Genomic and coexpression analyses predict multiple genes involved in triterpene saponin biosynthesis in Medicago Truncatula. Plant Cell 2010, 22, 850-866. [CrossRef] [PubMed]

4. Jiang, B.Q.; Qu, H.H.; Kong, H.; Zhang, Y.; Liu, S.C.; Cheng, J.J.; Yan, X.; Zhao, Y. The Effects of sweet foods on the pharmacokinetics of glycyrrhizic acid by iceELISA. Molecules 2017, 22, 498. [CrossRef] [PubMed]

5. Moses, T.; Pollier, J.; Almagro, L.; Buyst, D.; Van, M.M.; Pedreño, M.A.; Martins, J.C.; Thevelein, J.M.; Goossens, A. Combinatorial biosynthesis of sapogenins and saponins in Saccharomyces cerevisiae using a C-16 $\alpha$ hydroxylase from Bupleurum Falcatum. Proc. Natl. Acad. Sci. USA 2014, 111, 1634-1639. [CrossRef] [PubMed]

6. Wei, W.; Wang, P.P.; Wei, Y.J.; Liu, Q.F.; Yang, C.S.; Zhao, G.P.; Yue, J.M.; Yan, X.; Zhou, Z.Z. Characterization of Panax ginseng UDP-Glycosyltransferases catalyzing protopanaxatriol and biosyntheses of bioactive ginsenosides F1 and Rh1 in metabolically engineered yeasts. Mol. Plant 2015, 8, 1412-1424. [CrossRef] [PubMed]

7. Zhang, J.S.; Dai, L.H.; Yang, J.G.; Liu, C.; Men, Y.; Zeng, Y.; Cai, Y.; Zhu, Y.M.; Sun, Y.X. Oxidation of cucurbitadienol catalyzed by CYP87D18 in the biosynthesis of mogrosides from Siraitia Grosvenorii. Plant Cell Physiol. 2016, 57, 1000. [CrossRef]

8. Zhu, M.; Wang, C.X.; Sun, W.T.; Zhou, A.Q.; Wang, Y.; Zhang, G.L.; Zhou, X.H.; Huo, Y.X.; Li, C. Boosting 11 -oxo- $\beta$-amyrin and glycyrrhetinic acid synthesis in Saccharomyces cerevisiae via pairing novel oxidation and reduction system from legume plants. Metab. Eng. 2017, 45, 43. [CrossRef]

9. Li, L.; Shin, S.Y.; Lee, S.J.; Moon, J.S.; Im, W.T.; Han, N.S. Production of ginsenoside F2 by using Lactococcus lactis with enhanced expression of $\beta$-glucosidase gene from Paenibacillus Mucilaginosus. J. Agric. Food Chem. 2016, 64, 2506. [CrossRef]

10. Mochida, K.; Sakurai, T.; Seki, H.; Yoshida, T.; Takahagi, K.; Sawai, S.; Uchiyama, H.; Muranaka, T.; Saito, K. Draft genome assembly and annotation of Glycyrrhiza uralensis, a medicinal legume. Plant J. 2017, 89, 181-194. [CrossRef]

11. Wu, C.H.; Chen, A.Z.; Yen, G.C. Protective effects of glycyrrhizic acid and $18 \beta$-glycyrrhetinic acid against cisplatin-induced nephrotoxicity in BALB/c mice. J. Agric. Food Chem. 2015. [CrossRef] [PubMed] 
12. Zhao, Y.J.; Lv, B.; Feng, X.D.; Li, C. A Perspective on biotransformation and de novo biosynthesis of licorice constituents. J. Agric. Food Chem. 2017, 65, 11147-11156. [CrossRef] [PubMed]

13. Li, G.; Nikolic, D.; van Breemen, R.B. Identification and chemical standardization of licorice raw materials and dietary supplements using UHPLC-MS/MS. J. Agric. Food Chem. 2016, 64, 8062-8070. [CrossRef] [PubMed]

14. Asl, M.N.; Hosseinzadeh, H. Review of pharmacological effects of Glycyrrhiza sp. and its bioactive compounds. Phytother. Res. 2008, 22, 709-724. [CrossRef] [PubMed]

15. Bordbar, N.; Karimi, M.H.; Amirghofran, Z. The effect of glycyrrhizin on maturation and T cell stimulating activity of dendritic cells. Cell. Immunol. 2012, 280, 44-49. [CrossRef] [PubMed]

16. Zhao, Y.J.; Li, C. Biosynthesis of plant triterpenoid saponins in microbial cell factories. J. Agric. Food Chem. 2018, 66, 12155-12165. [CrossRef]

17. Kim, J.S.; Cheon, S.; Kim, S.W.; Kim, B.; Kim, H.; Park, K.D.; Kim, S.M. Glycyrrhizic acid prevents astrocyte death by neuromyelitis optica-specific IgG via inhibition of C1q binding. Biochem. Biophys. Res. Commun. 2016, 478, 553-558. [CrossRef]

18. Zhang, G.L.; Cao, Q.; Liu, J.Z.; Liu, B.Y.; Li, J.; Li, C. Refactoring $\beta$-amyrin synthesis in Saccharomyces Cerevisiae. AIChE J. 2015, 61, 3172-3179. [CrossRef]

19. Chen, K.; He, J.; Hu, Z.; Song, W.; Yu, L.; Li, K.; Qiao, X.; Ye, M. Enzymatic glycosylation of oleanane-type triterpenoids. J. Asian Nat. Prod. Res. 2018, 1-9. [CrossRef]

20. He, J.B.; Chen, K.; Hu, Z.M.; Li, K.; Song, W.; Yu, L.Y.; Leung, C.H.; Ma, D.L.; Qiao, X.; Ye, M. UGT73F17, a new glycosyltransferase from Glycyrrhiza uralensis, catalyzes the regiospecific glycosylation of pentacyclic triterpenoids. Chem. Commun. 2018. [CrossRef]

21. Xu, G.J.; Cai, W.; Gao, W.; Liu, C.S. A novel glucuronosyltransferase has an unprecedented ability to catalyse continuous two-step glucuronosylation of glycyrrhetinic acid to yield glycyrrhizin. New Phytol. 2016, 212, 123. [CrossRef] [PubMed]

22. Liu, X.C.; Zhang, L.; Feng, X.D.; Lv, B.; Li, C. Biosynthesis of glycyrrhetinic acid-3-O-monoglucose using glycosyltransferase UGT73C11 from Barbar. Ind. Eng. Chem. Res. 2017, 56, 14949-14958. [CrossRef]

23. Chang, T.S.; Chiang, C.M.; Wang, T.Y.; Lee, C.H.; Lee, Y.W.; Wu, J.Y. New triterpenoid from novel triterpenoid 15-O-glycosylation on ganoderic acid A by intestinal bacteria of Zebrafish. Molecules 2018, 23, 2345. [CrossRef] [PubMed]

24. Dai, L.H.; Liu, C.; Li, J.; Dong, C.X.; Yang, J.G.; Dai, Z.B.; Zhang, X.L.; Sun, Y.X. One-pot synthesis of ginsenoside $\mathrm{Rh} 2$ and bioactive unnatural ginsenoside by coupling promiscuous glycosyltransferase from Bacillus subtilis 168 to sucrose Synthase. J. Agric. Food Chem. 2018, 66. [CrossRef] [PubMed]

25. Dai, L.H.; Li, J.; Yang, J.G.; Zhu, Y.M.; Men, Y.; Zeng, Y.; Cai, Y.; Dong, C.X.; Dai, Z.B.; Zhang, X.L.; et al. Use of a promiscuous glycosyltransferase from Bacillus subtilis 168 for the enzymatic synthesis of novel protopanaxatriol-type ginsenosides. J. Agric. Food Chem. 2018, 66, 943-949. [CrossRef] [PubMed]

26. Dai, L.H.; Li, J.; Yao, P.Y.; Zhu, Y.M.; Men, Y.; Zeng, Y.; Yang, J.G.; Sun, Y.X. Exploiting the aglycon promiscuity of glycosyltransferase Bs-YjiC from Bacillus subtilis and its application in synthesis of glycosides. J. Biotechnol. 2017, 248, 69-76. [CrossRef] [PubMed]

27. Song, M.C.; Kim, E.; Ban, Y.H.; Yoo, Y.J.; Kim, E.J.; Park, S.R.; Pandey, R.P.; Sohng, J.K.; Yoon, Y.J. Achievements and impacts of glycosylation reactions involved in natural product biosynthesis in prokaryotes. Appl. Microbiol. Biotechnol. 2013, 97, 5691-5704. [CrossRef] [PubMed]

28. Hayashi, H.; Fukui, H.; Tabata, M. Biotransformation of $18 \beta$-glycyrrhetinic acid by cell suspension cultures of Glycyrrhiza Glabra. Phytochemistry 1990, 29, 2149-2152. [CrossRef]

29. Dai, L.H.; Liu, C.; Zhu, Y.M.; Zhang, J.S.; Men, Y.; Zeng, Y.; Sun, Y.X. Functional characterization of cucurbitadienol synthase and triterpene glycosyltransferase involved in biosynthesis of mogrosides from Siraitia Grosvenorii. Plant Cell Physiol. 2015, 56, 1172. [CrossRef]

30. Schmölzer, K.; Gutmann, A.; Diricks, M.; Desmet, T.; Nidetzky, B. Sucrose synthase: A unique glycosyltransferase for biocatalytic glycosylation process development. Biotechnol. Adv. 2015, 34, 88-111. [CrossRef]

31. Cinatl, J.; Morgenstern, B.; Bauer, G.; Chandra, P.; Rabenau, H.; Doerr, H.W. Glycyrrhizin, an active component of liquorice roots, and replication of SARS-associated coronavirus. Lancet 2003, 361, 2045. [CrossRef] 
32. Thibodeaux, C.J.; Melan, C.E.; Liu, H.W. Natural-product sugar biosynthesis and enzymatic glycodiversification. Angew. Chem. Int. Ed. 2009, 47, 9814-9859. [CrossRef] [PubMed]

33. Seki, H.; Ohyama, K.; Sawai, S.; Mizutani, M.; Ohnishi, T.; Sudo, H.; Akashi, T.; Aoki, T.; Saito, K.; Muranaka, T. Licorice $\beta$-amyrin 11-oxidase, a cytochrome $\mathrm{P} 450$ with a key role in the biosynthesis of the triterpene sweetener glycyrrhizin. Proc. Natl. Acad. Sci. USA 2008, 105, 14204. [CrossRef] [PubMed]

34. Seki, H.; Sawai, S.; Ohyama, K.; Mizutani, M.; Ohnishi, T.; Sudo, H.; Fukushima, E.O.; Akashi, T.; Aoki, T.; Saito, K. Triterpene functional genomics in licorice for identification of CYP72A154 involved in the biosynthesis of glycyrrhizin. Plant Cell 2011, 23, 4112-4123. [CrossRef] [PubMed]

35. Dewitte, G.; Walmagh, M.; Diricks, M.; Lepak, A.; Gutmann, A.; Nidetzky, B.; Desmet, T. Screening of recombinant glycosyltransferases reveals the broad acceptor specificity of stevia UGT-76G1. J. Biotechnol. 2016, 233, 49-55. [CrossRef] [PubMed]

36. Dymarska, D.; Janeczko, T.; Suslow, E.K. Glycosylation of 3-hydroxyflavone, 3-methoxyflavone, quercetin and baicalein in fungal cultures of the Genus Isaria. Molecules 2018, 23, 2477. [CrossRef] [PubMed]

37. Nam, S.H.; Kim, Y.M.; Walsh, M.K.; Wee, Y.J.; Yang, K.Y.; Ko, J.; Han, S.; Nguyen, T.T.H.; Ji, Y.K.; Kim, D. Synthesis and functional characterization of caffeic acid glucoside using Leuconostoc mesenteroides dextransucrase. J. Agric. Food Chem. 2017, 65. [CrossRef] [PubMed]

38. Winter, K.D.; Dewitte, G.; Dirkshofmeister, M.E.; Laet, S.D.; Pelantová, H.; Kren, V.; Desmet, T. Enzymatic glycosylation of phenolic antioxidants: Phosphorylase mediated synthesis and characterization. J. Agric. Food Chem. 2015, 63, 10131-10139. [CrossRef] [PubMed]

39. Bruyn, F.D.; Maertens, J.; Beauprez, J.; Soetaert, W.; Mey, M.D. Biotechnological advances in UDP-sugar based glycosylation of small molecules. Biotechnol. Adv. 2015, 33, 288-302. [CrossRef]

40. Guo, L.C.; Katiyo, W.; Lu, L.S.; Zhang, X.; Wang, M.M.; Yan, J.A.; Mao, X.Y.; Yang, R.J.; Zou, L.; Zhao, W. Glycyrrhetic acid 3-O-mono- $\beta$-D-glucuronide (GAMG): An innovative high-potency sweetener with improved biological activities. Compr. Rev. Food Sci. Food Saf. 2018. [CrossRef]

41. Chen, J.Y.; Kaleem, I.; He, D.M.; Liu, G.Y.; Li, C. Efficient production of glycyrrhetic acid 3-O-mono- $\beta$-D-glucuronide by whole-cell biocatalysis in an ionic liquid/buffer biphasic system. Process. Biochem. 2012, 47, 908-913. [CrossRef]

42. Lepak, A.; Gutmann, A.; Kulmer, S.T.; Nidetzky, B. Creating a water-soluble resveratrol-based antioxidant by site-selective enzymatic glucosylation. ChemBioChem 2015, 16, 1870-1874. [CrossRef] [PubMed]

43. Liu, C.; Zeng, Y.; Dai, L.H.; Cai, T.Y.; Zhu, Y.M.; Dou, D.Q.; Ma, L.Q.; Sun, Y.X. Mogrol represents a novel leukemia therapeutic, via ERK and STAT3 inhibition. Am. J. Cancer Res. 2015, 5, 1308. [PubMed] 